\title{
Single-Cell RNA Sequencing Findings Encourage the Application of Three-Dimensional in Vitro Cell Co- Culture to Breast Cancer Research
}

\section{Shuqing Yang}

First People's Hospital of Foshan

\section{Peixian Chen}

First People's Hospital of Foshan

\section{Xiaofan Mao}

First People's Hospital of Foshan

KaiRong Lin

First People's Hospital of Foshan

Wei Li

First People's Hospital of Foshan

\section{Tiancheng He}

First People's Hospital of Foshan

\section{Huiqi Huang}

First People's Hospital of Foshan

\section{Guangyu Yao}

Southern Medical University Nanfang Hospital

\section{AiGuo Wu}

Zhujiang Hospital

Wei Luo

First People's Hospital of Foshan

Dan Zhou ( $\square$ zdan14@fsyyy.com )

First People's Hospital of Foshan https://orcid.org/0000-0001-8698-2196

\section{Guolin Ye}

First People's Hospital of Foshan

\section{Short report}

Keywords: three-dimensional cell co-culture, Single-cell RNA sequencing, MCF-7, breast cancer

Posted Date: July 6th, 2021

DOI: https://doi.org/10.21203/rs.3.rs-634121/v1 
License: (c) (i) This work is licensed under a Creative Commons Attribution 4.0 International License. Read Full License 


\section{Abstract}

Traditional research on breast cancer based on cell lines has its limitations. Three-dimensional (3D) cell co-culture is an important tool for breast cancer research. However, while researchers wait for single-cell RNA sequencing technology to mature, it remains impossible to study the 3D cell co-culture cellular function accurately at single-cell levels. In this study, MCF-7, HMF, and HUVEC cells were mixed and cultured directly. The transcriptome changes after cisplatin intervention were detected by single-cell RNA sequencing. Our work aims to verify the advantages of $3 \mathrm{D}$ cell co-culture, which seems set to replace the traditional cell line experiment and become a part of mainstream cancer cell in vitro experiments.

\section{Introduction}

Using cell lines to study the biological characteristics of tumors is a common cytological experiment. However, the traditional two-dimensional cell line experiment cannot fully reveal the complexity of the tumor in vivo. Cell co-culture experiments (e.g., using a Transwell incubator) can be used to study cell interaction. However, these cell co-culture approaches do not involve direct contact between cells but rather communication between extracellular vesicles and other factors[1,2]. The latest research in this field shows that the growth of tumor cells in three-dimensional (3D) culture can maintain cell heterogeneity, especially the co-culture of multiple cells, which can better reflect the real situation of tumors than two-dimensional culture[3]. At present, due to advances in single-cell sequencing technology, we can directly use co-culture cells and then identify different cells at the single-cell levels[4].

In this study, MCF-7, HMF, and HUVEC were mixed for 3D culture and then treated with cisplatin to observe the transcription changes of MCF-7 at the single-cell level. The purpose of this study was to prove that 3D cell culture - with the help of single-cell RNA sequencing technology - will be a reliable and feasible method for tumor research.

\section{Method}

\section{Three-dimensional in vitro cell co-culture}

The experimental samples were divided into four groups: cell co-culture control group, cell co-culture cisplatin-induced group, MCF-7 cultured alone group, and MCF-7 cultured alone cisplatin-induced group. For cell co-culture, MCF-7, HMF, and HUVEC were mixed at a ratio of 1:1:1. All the cells were cultured in the previously prepared Matrigel matrix. The cell culture medium was dmem-h supplemented with $15 \%$ FBS, $1 \%$ dual antibody. The cells in the co-culture cisplatin-induced group and the MCF-7 alone cisplatininduced group were incubated with cisplatin at a concentration of $1.25 \mathrm{~mm} / \mathrm{ml}$ after 7 days of cisplatin treatment. The cells were harvested after 2 days of cisplatin treatment and subjected to single-cell RNA sequencing.

\section{Processing and analysis of single-cell RNA-seq data}


The single-cell suspension was prepared, and cDNA was synthesized by BD Rhapsody Express single-cell analysis system. The final library was quantified by Qubit fluorescence assay using Qubit dsDNA HS kit (ThermoFisher). In the laboratory of Novogene Biotech Co., Ltd (Beijing, China), the library was sequenced on paired NovaSeq end 6000 by paired-end mode library. R 3.6.2 was used to process raw readings scRNA-seq, and the R/Seurat package was used to analyze gene expression data. We excluded cells expressing less than 300 genes and cells expressing more than 6000 genes. To eliminate dying cells or low-quality cells with extensive mitochondrial pollution, we also discarded those cells where mitochondrial genes accounted for over than $30 \%$ of the signal. Subsequently, $t$ distribution random neighborhood embedding (tSNE) was applied to the two-dimensional representation of data structure. After clustering, the subtypes of co-cultured cells were identified by R package SingleR[5], and MCF-7, HMF, and HUVEC cells were identified from co-cultured cells. After completing the bioinformatics analysis of each group of differential genes, we input the differential express genes into CancerSEA (http://biocc.hrbmu.edu.cn/) to check the functional analysis of the differential express genes in the single-cell sequencing database.

\section{Results}

\section{D cell culture and identification Groups}

In the process of 3D cell culture, we found that the cell clusters formed by the co-culture of the three types of cells are smoother and more regular, while the cell clusters formed on Matrigel by simple MCF-7 have different sizes and irregular shapes(Figure 1A-F). We performed scRNA-seq on the 3D culture cells(Figure $1 \mathrm{G})$, and after quality control, approximately 7088 cells met quality control metrics and were analyzed. Unsupervised clustering using the Seurat package identified six clusters of cells (cluster $0-5$, Figure $1 \mathrm{H}, \mathrm{I}$ ). Cluster 0,1 , and 3 were classified as HUVEC, Cluster 2 and 4 were classified as MCF-7, and Cluster 5 was classified as HMF(Figure 2A-B). We also screened the highly expressed genes of each group of cells, among which the HUVEC highly expressed genes included C15orf48, PLAU, CCL20, PDZK1IP1, and MT2A(Figure 2C-D). The MCF-7 highly expressed genes include TXNIP, COX6C, CRABP2, CCN5, CLDN4, TFF1, KRT19(Figure 2G-H), and HMFs' highly expressed genes include MMP2, COL6A1, SPARC, DCN, and COL1A1(Figure 2E-F).

\section{MCF-7 response after cisplatin stimulation at single-cell levels.}

The purpose of this study was to compare the level of the single-cell MCF-7 cisplatin difference in response to the co-culture cultured alone. First, our results show that the transcriptomes of the two different states are very different (Figure 3A, B), and the difference between the two groups after cisplatin intervention is more significant (Figure 3C, D). However, there are also differences between the cisplatin intervention group and the control group under the mode of cell co-culture, but this difference is relatively mild. The highly expressed genes in MCF-7 after cisplatin intervention include HSPA8, HSPA1A, HSPA1B, FOS, STC1, LOXL2, CCN5, and MT2A, and the low-expressed genes include EIF5B, GDF15, USP8, XBP1, KRT18, and KRT19(Figure 4A). Next, we have performed signal pathway enrichment analysis and GO 
analysis of differential genes. The enrichment signal pathway of differential genes includes Protein processing in the endoplasmic reticulum, Antigen processing and presentation, and Estrogen signaling pathway(Figure 4A). CancerSEA is a specialized database aimed at single-cell resolution decoding comprehensive cancer cells' different functional states[6]. Finally, we analyze the differential genes and single-cell sequencing databases to further analyze the functions of the differentially expressed genes. The signal pathways enriched by these differential genes include Apoptosis, DNA damage, Hypoxia, and Metastasis, among others(Figure 4D-E).

\section{Discussion}

Modeling tumors in the laboratory is essential for improving understanding of their biology and developing new treatment strategies to treat breast cancer. At present, researchers have developed methods for culturing cancer cells in 3D, including lung cancer and liver cancer, which can better mimic the biological behavior of the in vivo tumor [7-9]. Before single-cell RNA sequencing technology was widely applied, immunohistochemistry was the main experimental method for exploring the pathogenesis of 3D cell co-culture or tissue culture [10]. However, immunohistochemistry could not identify and analyze RNA changes in a high-throughput way at the single-cell level.

In this study, the transcriptome of MCF-7 in co-cultured samples was significantly different from that of MCF-7 alone at the single-cell level. GO analysis and KEGG analysis of differentially expressed genes are similar to those reported in the literature, indicating that cell co-culture can change the characteristics and functions of MCF-7, which resembles the real pathophysiological situation in vivo. To further analyze the function of differentially expressed genes, we mapped the differential gene analysis to the breast cancerrelated single-cell sequencing database. The differentially expressed genes were enriched in apoptosis, DNA damage, hypoxia, and metastasis, which is consistent with the theoretical expectation.

The limitations of this study are that we co-cultured only cell types, without using immune cells, for example. Furthermore, at the end of the experiment, the proportion of HMF was relatively low.

The results of this study suggest that multicellular direct culture can be done. Then, data analysis based on single-cell sequencing technology can overcome the shortcomings of bulk sequencing, which cannot identify cell subsets for the co-cultured study. Therefore, conclusions drawn from this type of experiment should be closer to the truth of the tumor than those based on cell line studies.

\section{Declarations}

\section{Ethics approval and consent to participate}

Not applicable

\section{Consent for publication}


Not applicable

\section{Competing interests}

The authors declare that they have no competing interests.

\section{Funding}

This study was funded by the National Natural Science Foundation of China (grant numbers. 81972335 and 81701817); Guangdong Medical Research Fund (grant number. A2019329).

\section{Authors' contributions}

DZ and WL conceived and designed the experiments. WL, PXC and SQY performed the experiments. TCH and XFM analyzed the data. KRL and HQH searched the literature. GYY, GLY and AGW drafted the manuscript. All authors read and approved the final manuscript.

\section{Acknowledgements}

The authors would like to thank Guangzhou GenCoding Co., Ltd for their technical support in this study.

\section{Availability of data and materials}

The datasets of the current study are available from the corresponding authors.

\section{References}

1. Vis MAM, Ito K, Hofmann S. Impact of Culture Medium on Cellular Interactions in in vitro Co-culture Systems. Front Bioeng Biotechnol. 2020;8:911. http://doi.org/10.3389/fbioe.2020.00911.

2. Thayanithy V, O'Hare P, Wong P, Zhao X, Steer CJ, Subramanian S, et al. A transwell assay that excludes exosomes for assessment of tunneling nanotube-mediated intercellular communication. Cell communication signaling: CCS. 2017;15(1):46. http://doi.org/10.1186/s12964-017-0201-2.

3. Langhans SA. Three-Dimensional in Vitro Cell Culture Models in Drug Discovery and Drug Repositioning. Front Pharmacol. 2018;9:6. http://doi.org/10. 3389/fphar.2018.00006.

4. Chen YC, Jung S, Zhang Z, Wicha MS, Yoon E. Co-culture of functionally enriched cancer stem-like cells and cancer-associated fibroblasts for single-cell whole transcriptome analysis. Integrative biology: quantitative biosciences from nano to macro. 2019;11(9):353-61. http://doi.org/10.1093/intbio/zyz029.

5. Aran D, Looney AP, Liu L, Wu E, Fong V, Hsu A, et al. Reference-based analysis of lung single-cell sequencing reveals a transitional profibrotic macrophage. Nature immunology. 2019;20(2):163-72. http://doi.org/10.1038/s41590-018-0276-y.

6. Yuan H, Yan M, Zhang G, Liu W, Deng C, Liao G, et al. CancerSEA: a cancer single-cell state atlas. Nucleic acids research. 2019;47(D1):D900-D8. http://doi.org/ 10.1093/nar/gky939. 
7. Chaicharoenaudomrung $N$, Kunhorm $P$, Noisa P. Three-dimensional cell culture systems as an in vitro platform for cancer and stem cell modeling. World journal of stem cells. 2019;11(12):1065-83. http://doi.org/10.4252/wjsc.v11.i12.1065.

8. Takai A, Fako V, Dang H, Forgues M, Yu Z, Budhu A, et al. Three-dimensional Organotypic Culture Models of Human Hepatocellular Carcinoma. Scientific reports. 2016;6:21174. http://doi.org/10.1038/srep21174.

9. Ziolkowska-Suchanek I. Mimicking Tumor Hypoxia in Non-Small Cell Lung Cancer Employing ThreeDimensional Vitro Models Cells. 2021;10(1):141. http://doi. org/10.3390/cells10010141.

10. Wagner I, Materne EM, Brincker S, Sussbier U, Fradrich C, Busek M, et al. A dynamic multi-organ-chip for long-term cultivation and substance testing proven by 3D human liver and skin tissue co-culture. Lab Chip. 2013;13(18):3538-47. http://doi.org/10.1039/c3lc50234a.

\section{Figures}


A

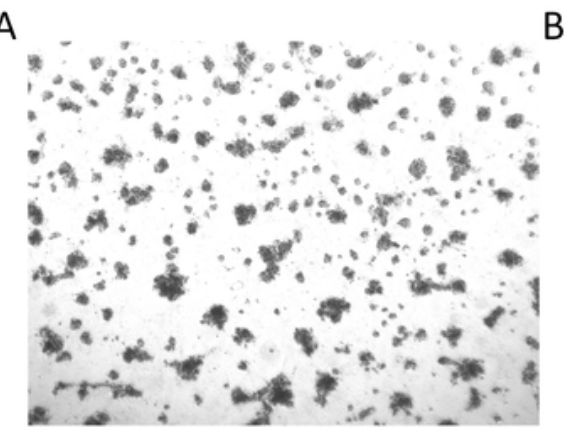

D

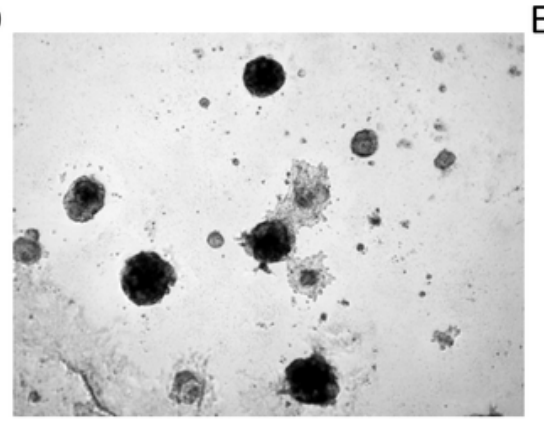

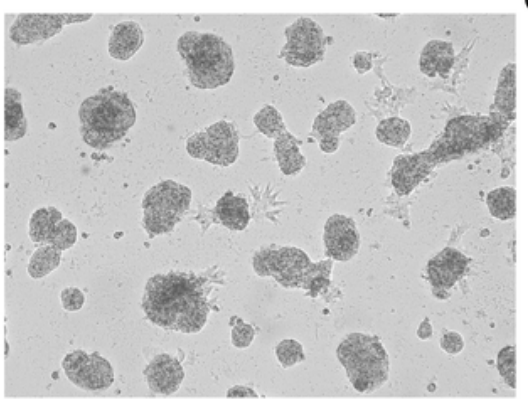

E

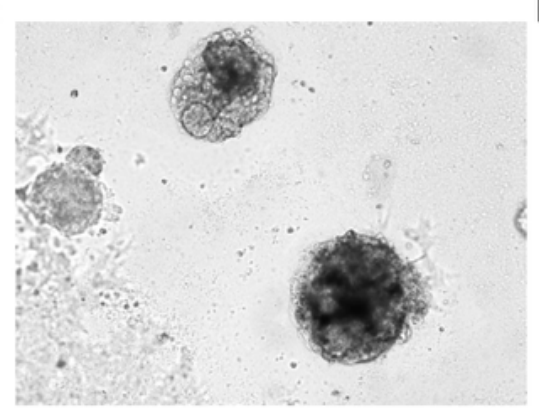

C

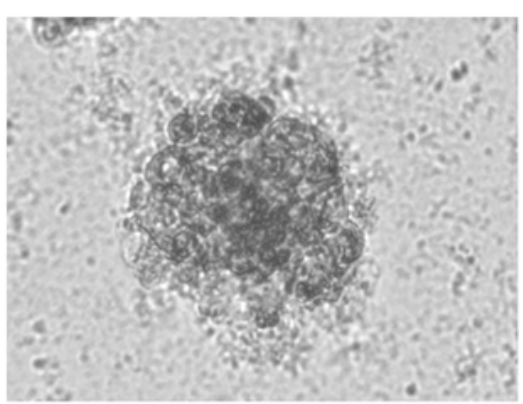

F

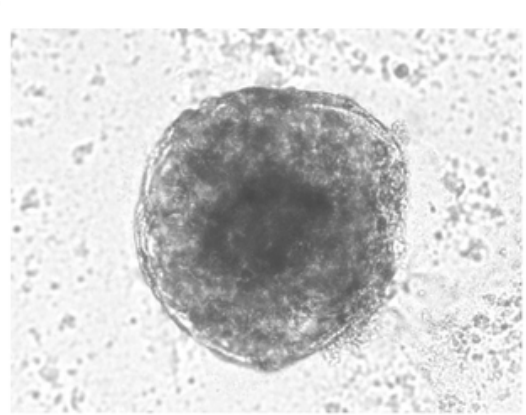

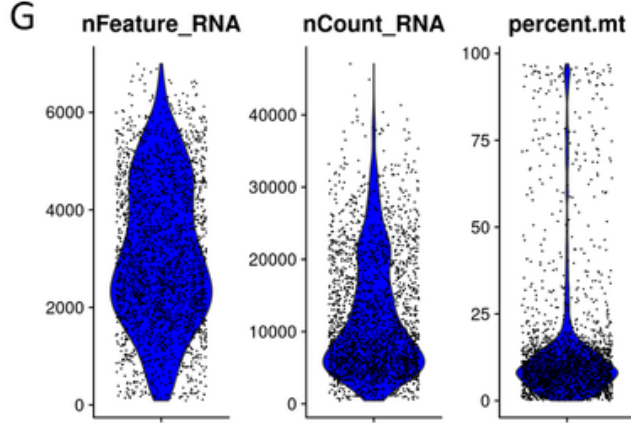

$\mathrm{H}$

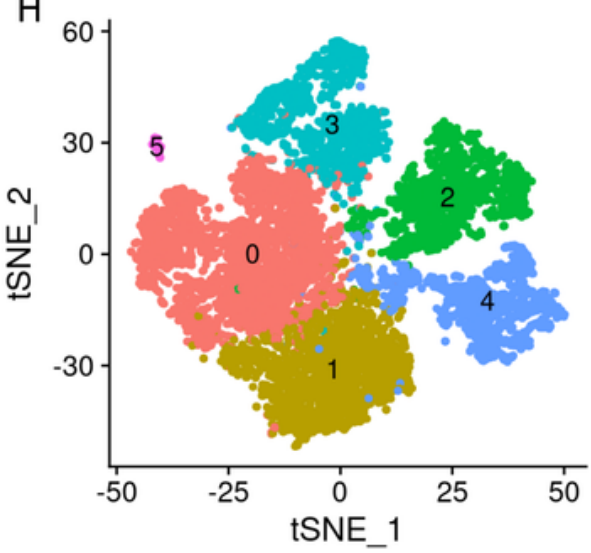

MC_HM_HU_Matrigel_Control

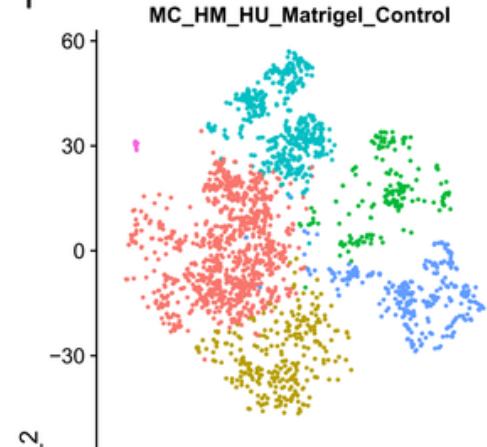

岁

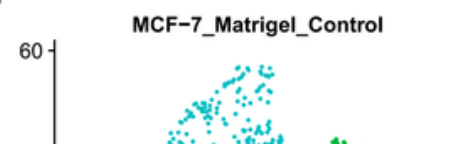

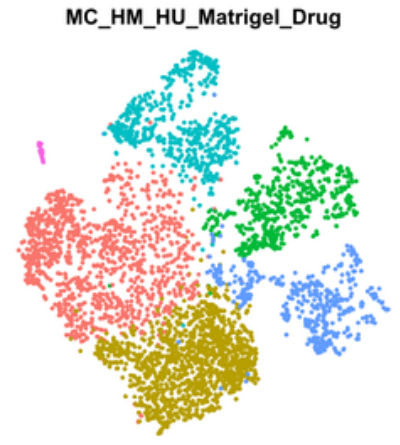

MCF-7_Matrigel_Drug

six

in.

a

a

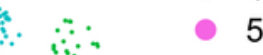

- 0

- 1

- 2

- 3

- 4

- 5

\section{Figure 1}

3D cell culture and cell-type identification. A-C, MCF-7 cells cultured alone, cell spheres were irregular. DF, Co-cultured cells, formed a 3D structure with a smooth edge. G, Single-cell RNA sequencing data showed the number of RNAs, the number of counts, and the proportion of mitochondrial RNAs. 1. Cluster graph of four samples. $\mathrm{H}$. The cluster diagram of all cells shows that they can be divided into six clusters. 


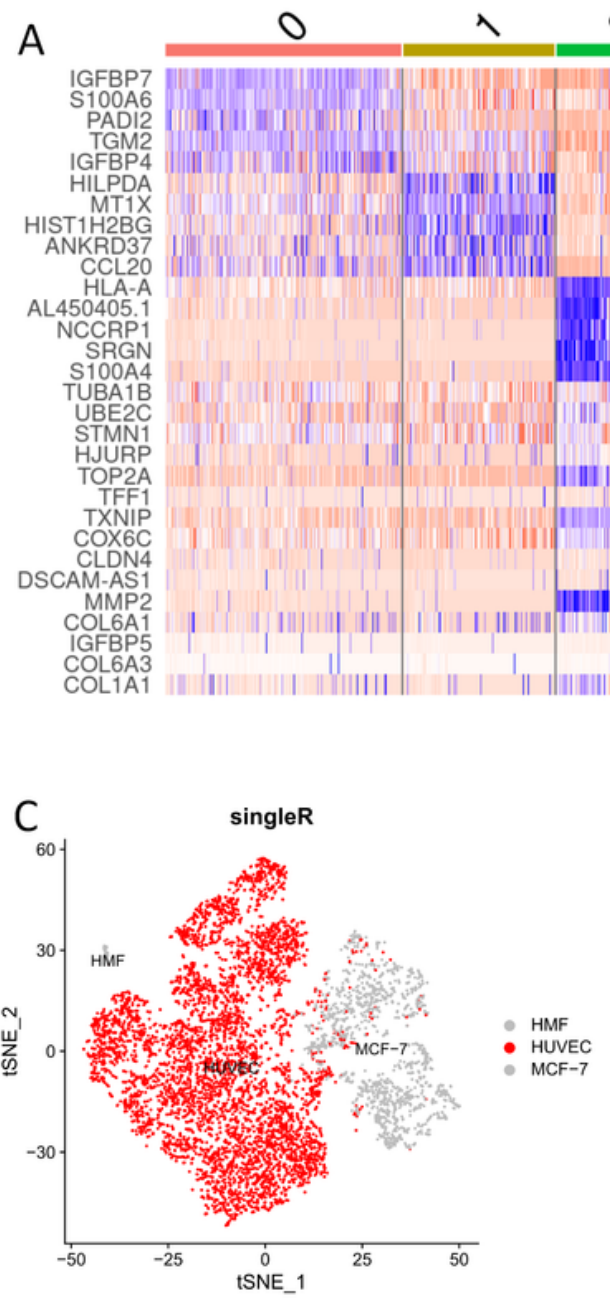

$23 \propto 5$

Identity

B

singleR

- 1

- 2

- 3

- 4

- 5

Expression
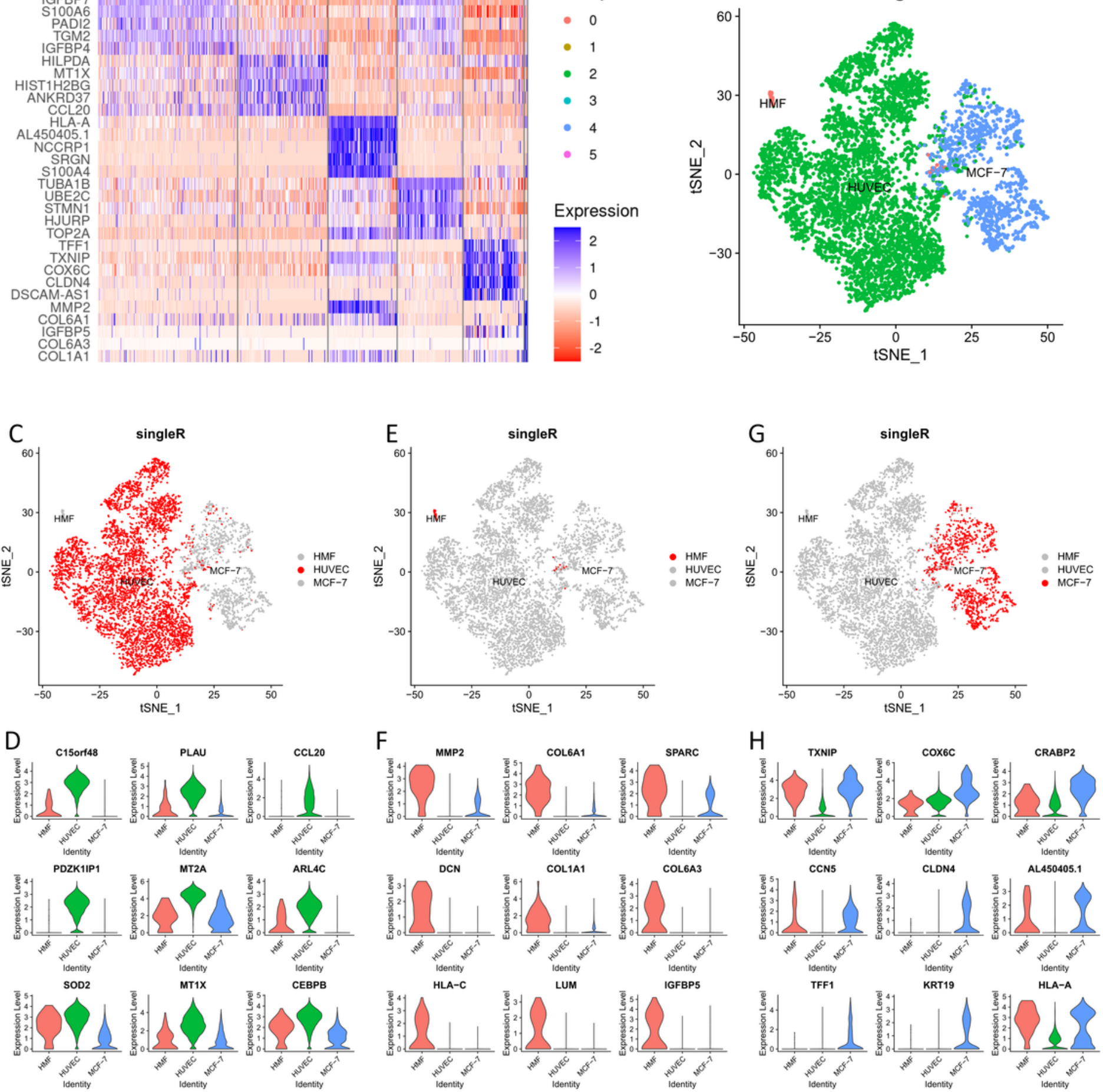

Figure 2

The RNA characteristics of cells were analyzed at the single-cell level. A, The characteristic gene heatmap of bases on clusters. B, Cluster identification, which is mainly composed of HUVEC and MCF-7 cells, HMF accounted for a small proportion. $C$ and $D, H U V E C$ and its characteristic genes. $E$ and F, HMF and its characteristic genes. G and H, MCF-7 and its characteristic genes. 

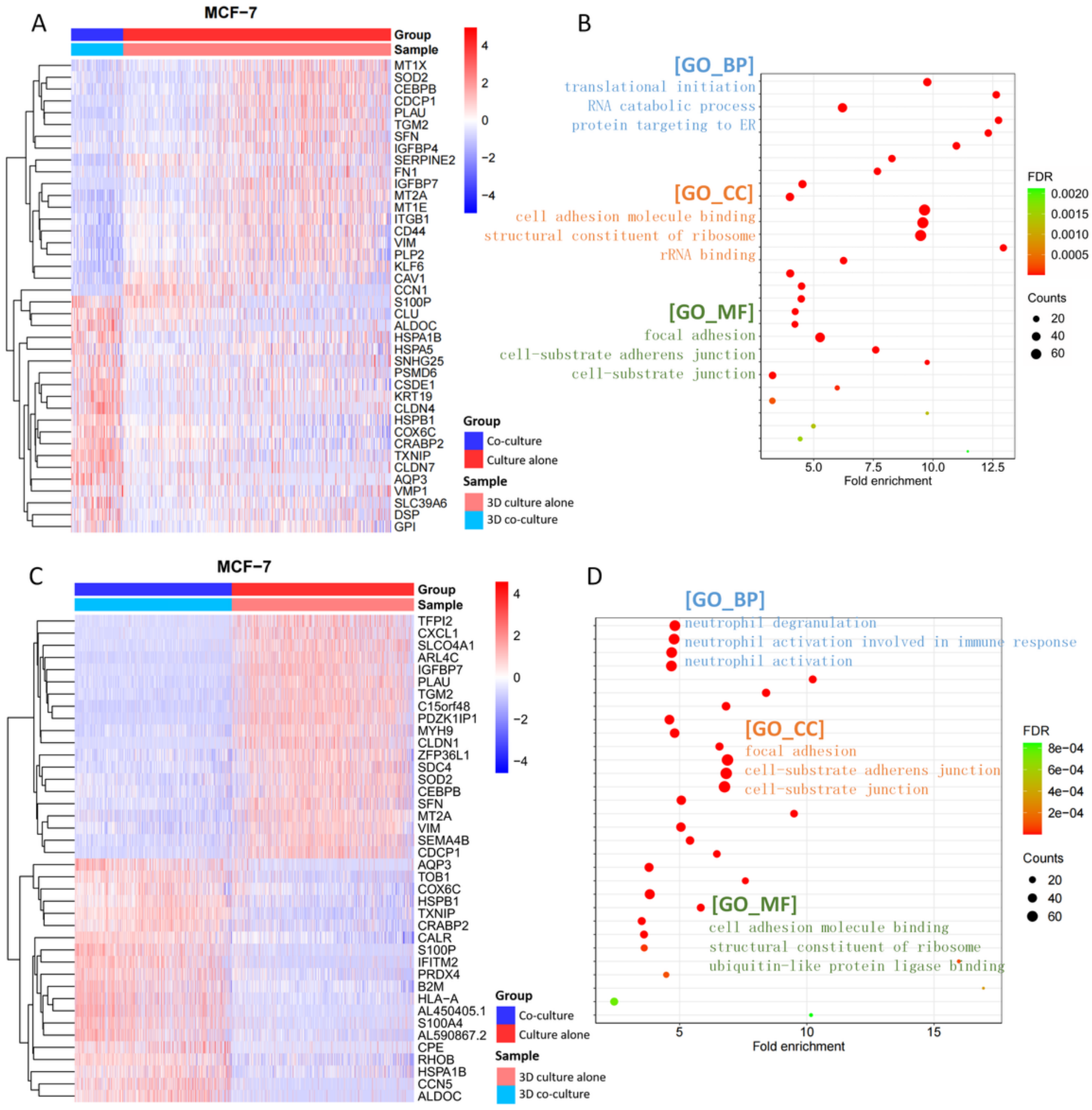

Figure 3

The RNA expression of MCF-7 in different groups. A and B, The heatmap of differential gene cluster analysis and GO enrichment analysis of co-culture and culture alone of MCF-7 cells. C and D, Heatmap of the differential gene cluster analysis and GO enrichment analysis of cisplatin response of MCF-7 cell coculture and culture alone. 
A

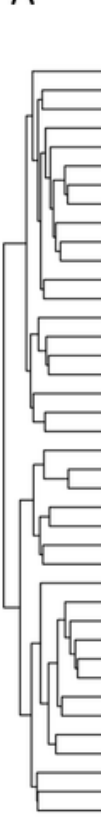

C

\begin{tabular}{clcc}
\multicolumn{1}{c}{ ID } & \multicolumn{1}{c}{ Description } & Counts & P Value \\
hsa04141 & Protein processing in endoplasmic reticulum & 14 & $4.04 \mathrm{E}-10$ \\
hsa04612 & Antigen processing and presentation & 10 & $5.31 \mathrm{E}-10$ \\
hsa04915 & Estrogen signaling pathway & 10 & $4.68 \mathrm{E}-07$ \\
hsa05167 & Kaposi sarcoma-associated herpesvirus infection & 10 & $1.07 \mathrm{E}-05$ \\
hsa05169 & Epstein-Barr virus infection & 10 & $1.40 \mathrm{E}-05$ \\
hsa05170 & Human immunodeficiency virus 1 infection & 10 & $2.15 \mathrm{E}-05$ \\
hsa05417 & Lipid and atherosclerosis & 10 & $2.44 \mathrm{E}-05$ \\
hsa04218 & Cellular senescence & 8 & $9.31 \mathrm{E}-05$ \\
hsa05418 & Fluid shear stress and atherosclerosis & 7 & 0.00026 \\
hsa05012 & Parkinson disease & 9 & 0.00047
\end{tabular}

B

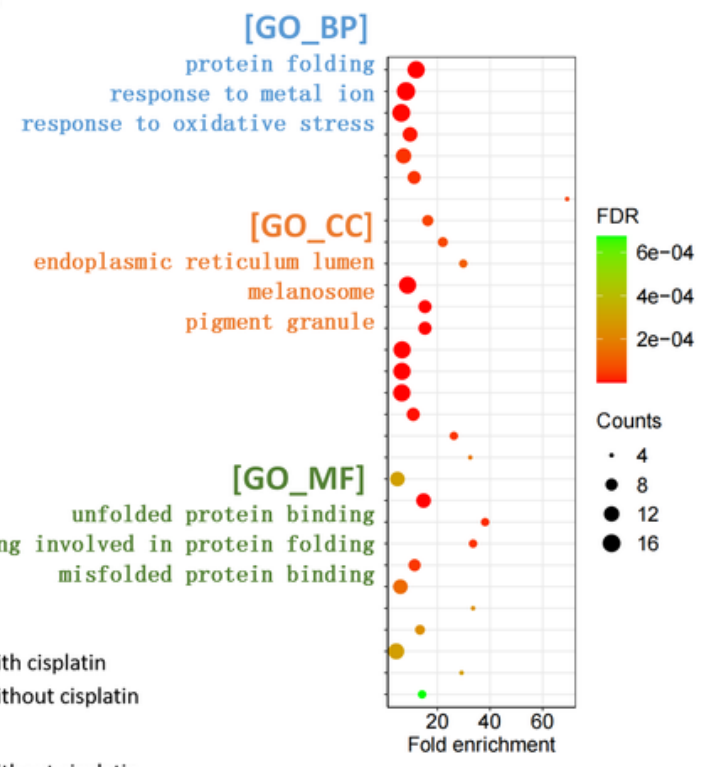

E Expression distribution T-SNE plot
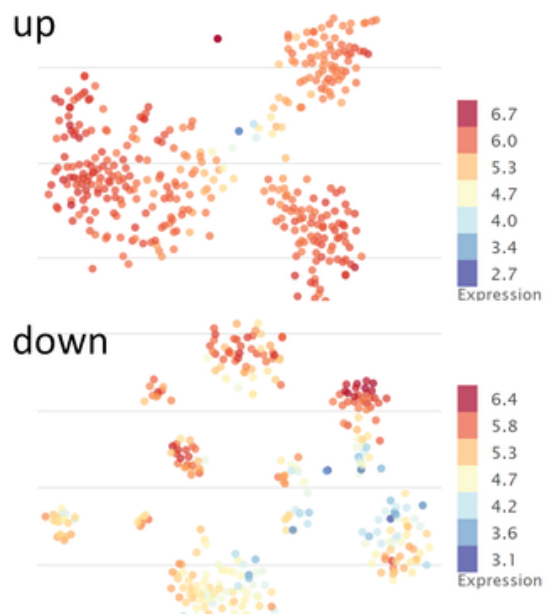

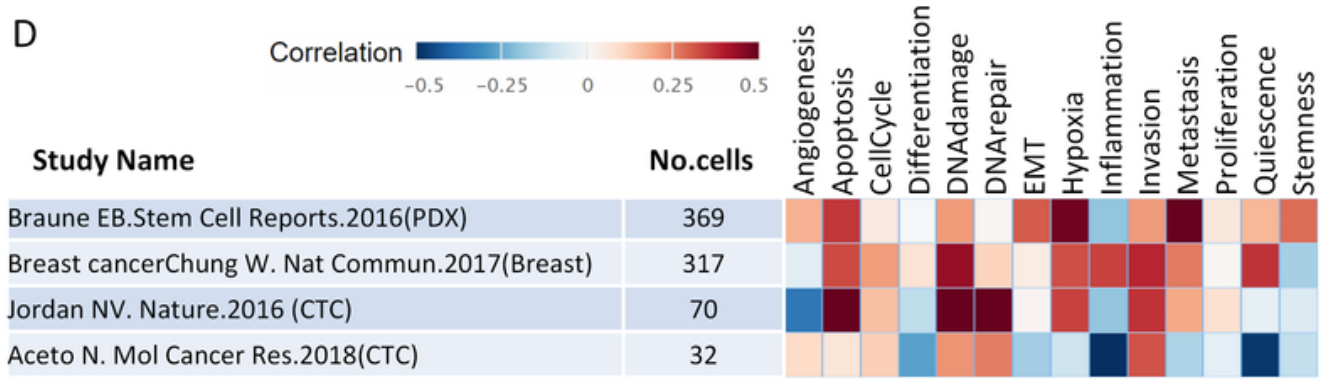

\section{Figure 4}

Cisplatin response of MCF-7 in 3D cell co-culture. A, Heatmap of the differential gene of MCF-7 before and after cisplatin treatment. B, GO enrichment analysis of differential gene. C, KEGG analysis of differential gene. D, Mapping of differentially expressed genes in CancerSEA, the up is derived from the studs of breast cancer. The box diagram indicates the distribution of gene expression levels in cells of the dataset. T-SNE describes the distribution of cells, every point represents a single cell, and the color of the 
point represents the expression level of the gene in the cell. E, Functional correlation analysis of differential genes was performed in four different data sets that form the CancerSEA. 